Cinémas

Revue d'études cinématographiques

Journal of Film Studies

\title{
La réécriture comme plus-value sémantique : montage, technique et discours dans le cinéma africain
}

\section{Alexie Tcheuyap}

Volume 11, numéro 1, automne 2000

Écritures dans les cinémas d'Afrique noire

URI : https://id.erudit.org/iderudit/024835ar

DOI : https://doi.org/10.7202/024835ar

Aller au sommaire du numéro

Éditeur(s)

Cinémas

ISSN

1181-6945 (imprimé)

1705-6500 (numérique)

Découvrir la revue

Citer cet article

Tcheuyap, A. (2000). La réécriture comme plus-value sémantique : montage, technique et discours dans le cinéma africain. Cinémas, 11(1), 77-95.

https://doi.org/10.7202/024835ar
Résumé de l'article

Un fait semble avoir jusqu'ici échappé à la recherche : la capacité d'emprunt du cinéma africain. Nombre de films produits sur le continent sont en effet tirés de textes littéraires. Cet article engage de manière frontale la question des réécritures filmiques en Afrique. Que devient le texte littéraire à l'écran? En admettant que le texte est ouvert à toute hypothèse herméneutique, quels sens sont ajoutés au texte littéraire par le film? L'analyse considère deux prismes majeurs : le montage et la technique comme lieux d'inscription discursive permettant de porter le film à des horizons sémantiques inexplorés par le texte tuteur. On se situe dès lors dans le domaine de renonciation dont on déterminera des signifiants spécifiques. 


\title{
La réécriture comme plus-value sémantique: montage, technique et discours dans le cinéma africain
}

\section{Alexie Tcheuyap}

\begin{abstract}
RÉSUMÉ
Un fait semble avoir jusqu'ici échappé à la recherche: la capacité d'emprunt du cinéma africain. Nombre de films produits sur le continent sont en effet tirés de textes littéraires. Cet article engage de manière frontale la question des réécritures filmiques en Afrique. Que devient le texte littéraire à l'écran? En admettant que le texte est ouvert à toute hypothèse herméneutique, quels sens sont ajoutés au texte littéraire par le film? Lanalyse considère deux prismes majeurs: le montage et la technique comme lieux d'inscription discursive permettant de porter le film à des horizons sémantiques inexplorés par le texte tuteur. On se situe dès lors dans le domaine de l'énonciation dont on déterminera des signifiants spécifiques.
\end{abstract}

\begin{abstract}
A fact seems to have eluded research so far: the capacity of African cinema to borrow. Many films produced on that continent have indeed been adapted from literary texts. This article deals head-on with the question of film réecritures in Africa. What becomes of the literary text on the screen? Assuming that the text is open to any hermeneutic hypothesis, which meanings are added to the literary text by the film? This analysis takes into account two major aspects: montage and technique, as loci of discursive inscription allowing the film to be brought to semantic horizons unexplored by the source text. It then situates itself in the domain of
\end{abstract}


enunciation, for which specific signifiers will be identified.

Lorsque Émile Benveniste (1966) définit l'objectivité du récit par rapport au discours, cette objectivité, il le souligne, est toute relative. Elle est construite et est un leurre. De même, au cinéma, Christian Metz (1977, p. 113) soutient que:

[1]e film traditionnel se donne comme histoire, non comme discours. Il est pourtant discours, si l'on se réfere aux intentions du cinéaste, aux influences qu'il exerce sur le public, etc. Mais le propre de ce discourslà, le principe même de son efficace comme discours est justement d'effacer les marques d'énonciation et de se déguiser en histoire.

Pour explorer les modes d'inscription discursive du cinéma africain, on traitera ici exclusivement de deux aspects qu'on limitera aux réécritures filmiques: le montage et l'appareillage technique, lesquels engagent le texte sur un ensemble de réseaux interprétatifs inapparents. Depuis au moins les études de JeanLouis Comolli (1971), il est entendu que la technique, et particulièrement la caméra, n'est pas neutre. Elle est le lieu d'une inscription qu'il est possible de déterminer. Bien plus, le montage, en tant que violence faite au réel, n'est pas seulement une planification consécutive à un calcul. Il est surtout une forme discrète de déploiement discursif, c'est-à-dire idéologique. S'il permet selon Michel Colin (1985, p. 33) de développer l'identification,

[...] il doit aussi être défini comme permettant au film de se faire discours. Le montage a, en effet, pour fonction de mettre en relation des énoncés complets. Il ne peut donc pas simplement être défini comme syntaxe minimum des effets idéologiques de forme empirique, mais aussi comme organisation syntaxique du film comme discours idéologique spéculatif [...].

En considérant Sarraounia (1986) de Med Hondo, Xala (1974) et Guelwaar (1992) d'Ousmane Sembène, L'Aventure ambiguë (1984) de Jacques Champreux, L'Enfant noir (1995) de Laurent Chevalier et Sango Malo (1991) de Bassek Ba Kobhio, quels sont les lieux possibles de discours? Quelles hypothèses 
peut-on émettre à partir des opérations de montage et de l'usage de la technique dans ces films? Quels sens additionnels sont apportés au texte romanesque? Ce sont là autant de questions qu'on analysera à partir de certains débuts et fins de films, ces lieux stratégiques de la narration.

\section{Débuts et fins des films : les thèses Sembène et Hondo}

Si le montage est d'abord agencement de plans et rapport entre eux, puis disposition de séquences, on pourrait se demander ce qui conditionne l'ordonnancement syntaxique d'un film. Autrement dit, pourquoi le film commence-t-il par telle séquence plutôt que par telle autre? Et peut-on en effectuer une lecture de pareille conjoncture?

En se posant ces questions, on se rend compte qu'un film comme celui de Med Hondo comporte, dès son ouverture, des éléments pouvant orienter la compréhension. La séquence liminaire qui déploie le défilement du générique est en fait un planséquence. Autrement dit, le temps réel y donne l'illusion de l'authenticité. Sur un carton, il est écrit: "Ce film est basé sur des faits authentiques qui se sont déroulés entre 1878 et 1899 au Niger actuel, "Le procès narratif engagé se situe du côté de l'Histoire, et de l'authentification. Le film est un « récit historique» auquel le générique sert d'introduction.

Les travellings d'accompagnement montrent des hommes croulant sous des cargaisons énormes. La longue file de porteurs est précédée de quelques officiers à cheval portant le drapeau français qui flotte triomphalement. Déjà la mise en scène est parlante: les porteurs sont au milieu du convoi de piétons, ce qui permet de protéger le butin colonial. Quelques Nègres les précèdent, servant de boucliers contre une attaque éventuelle. Ils ont moins de valeur que le fruit de diverses rapines écrasant les suivants. De plus, les images indiquent que le procès narratif qui s'engagera dans Sarraounia ne sera pas de tout repos, car leur architecture connote le western ou le film de guerre. Non pas à cause des armes que portent les soldats, mais uniquement par ce décor liminaire. Les images donnent un effet de surimpression causé par les vents de sable qui parcourent l'écran. Le cadre initial n'est pas celui d'une quiétude quelconque. À ce sujet, la 
préparation thématique mise en jeu par le son et les images ne sera pas démentie dans la suite du film. Dès le début, il est possible de procéder à des anticipations. Le spectateur, aidé en cela par la voix off qui succède au carton initial, peut d'emblée spéculer sur les enjeux mis en œuvre dans le film ainsi que sur les différents acteurs. Med Hondo l'énonce dès le début sans en avoir l'air. L'argument syntaxique est moteur, tel que le relevait Michel Foucault (1966, p. 25):

[...] on a beau dire ce qu'on voit, ce qu'on voit ne loge jamais dans ce qu'on dit, et on a beau faire voir, par des images, des métaphores, des comparaisons, ce qu'on est en train de dire, le lieu où elles resplendissent n'est pas celui que déploient les yeux, mais celui que définissent les successions de la syntaxe.

Cet ordonnancement est aussi à l'œuvre chez Ousmane Sembène dont les films, du point de vue chronologique et idéologique, rendent compte des conséquences historiques de l'expédition coloniale mise en scène par Med Hondo. Par sa seule disposition syntaxique, Xala énonce dès la première séquence la thèse qui émaille tout le roman: l'indépendance est, plus que l'affliction d'une farce juridique, un ensemble d'impuissances. Elle marque surtout la naissance d'un personnel parasite dans les pays colonisés, de groupuscules de jouisseurs à qui les maîtres d'hier jettent en pâture le patrimoine national.

En effet, dès la séquence liminaire, quelques individus, d'allure paysanne ou du genre clochard, sortent de la foule et gravissent fièrement les marches de l'immeuble de la chambre de commerce, filmé en contre-plongée. C'est l'amorce d'une ascension sociale qui est ainsi présentée. Le même mouvement de caméra les montre levant les mains, signe de victoire, à la suite de l'expulsion feinte des colons. Après le départ un peu caricatural des deux Français officiant à la chambre, les nouveaux occupants reviennent au plan suivant, engoncés dans des costumes, ceux-là mêmes qu'arboraient leurs prédécesseurs. Les foules qui pavoisent pour célébrer le départ des maîtres, lesquels reviennent pourtant bientôt, ignorent tout du cirque qui se joue à l'intérieur. Le nombre de personnes devant officier à la chambre a 
plus que doublé. De trois, les indigènes le portent à huit. Connaissant sans doute le milieu, Dupont, l'ancien président de la chambre, et ses collaborateurs réapparaissent après quelques instants avec des mallettes pleines d'argent. On peut observer quelques-uns des membres contemplant avec un bonheur mal contenu les billets de banque serrés dans ces mallettes. Paradoxalement, toutes ces images se succèdent pendant que la voix du nouveau président de la chambre tient un discours sur la " révolution " des indépendances et l'africanisation des cadres:

\begin{abstract}
Nous devons prendre ce qui est à nous, ce qui nous revient de droit. Nous devons contrôler notre industrie. Face à notre peuple, nous devons montrer que nous sommes capables comme tous les autres peuples du monde. Nous sommes des hommes d'affaires. Nous devons prendre toutes les directions, les banques comprises. Notre marche est irréversible. C'est l'aboutissement de notre lutte pour l'indépendance. [...] Nous optons pour le socialisme, le seul vrai socialisme, la voie africaine du socialisme, le socialisme à hauteur d'hommes. Notre indépendance est complète.
\end{abstract}

La synchronisation de l'image et du son est d'une ironie cingiante et marque l'abîme qui a toujours existé en Afrique entre le discours politique et son application. Cette appropriation, cette égalité avec "tous les autres peuples du monde" se déploient de manière précise pendant le déroulement du discours du nouveau président : des mallettes pleines d'argent sont distribuées à chacun des nouveaux membres. Le "socialisme" qui, sur le continent, fraie la voie à de nouveaux monstres, est pire que l'expression d'une oppression durable. Il est surtout le signe de la naissance d'une bourgeoisie parasitaire dont le but unique, mis en scène par Sembène, est l'enrichissement illicite et rapide.

Cette perspective nous situe, selon Frantz Fanon, dans le registre de toutes les nouvelles bourgeoisies surgies du colonialisme. La nationalisation est en fait un changement de la nomenklatura, par lequel le patrimoine national se retrouve enseveli dans les ambitions de quelques larbins du nouvel ordre impérial. La colonisation et ce qui lui succède n'étant rien moins qu'un pillage massif, la passation consiste en fait, nous dit Fanon, et on

La réécriture comme plus-value sémantique : montage, technique et discours... 
le voit avec le film de Sembène, à "nationaliser le vol de la nation" (1968, p. 16). Une fois de plus, l'Afrique s'illustre par sa capacité à singer, jusqu'au grotesque le plus tragique comme c'est le cas avec cette caricature du socialisme, les aspects uniquement pervers des restes idéologiques venus d'ailleurs. Ainsi, la bourgeoisie naissante en scène dans cette séquence liminaire est exactement le contrepoint de la bourgeoisie occidentale qui est moteur de progrès:

Ce rôle lucratif, cette fonction de gagne-petit, cette étroitesse de vues, cette absence d'ambition symbolisent l'incapacité de la bourgeoisie nationale à remplir son rôle historique de bourgeoisie. Laspect dynamique et pionnier, l'aspect inventeur et découvreur de monde que l'on trouve chez toute bourgeoisie est ici lamentablement absent. Au sein de la bourgeoisie nationale des pays coloniaux, l'esprit jouisseur domine. [...] Elle suit la bourgeoisie occidentale dans son aspect négatif et décadent sans avoir franchi les premières étapes d'exploration et d'invention qui sont en tout état de cause un acquis de cette bourgeoisie occidentale (Fanon, 1968, p. 98-99).

On comprend donc que dans Xala, pour célébrer «l'indépendance ", les membres de la chambre de commerce soient invités à une cérémonie de mariage. En lieu et place d'une liberté et d'un projet cohérent de société, le "départ " du colon a en fait amorcé une interminable ruée épicuriste fondée sur le sexe, l'argent et la nourriture. Avec le plaisir pour seule idéologie et seul projet social. C'est pourquoi Sembène traite dans Guelwaar d'un autre piège par lequel l'Occident tient l'Afrique comme la corde tient un pendu : l'aide alimentaire. Le principe est d'entretenir chez le Nègre non pas la pensée ou un esprit réel d'entreprise mais, ainsi qu'il le montre dans Xala, la jouissance. Il est important de le maintenir dans les chaînes du plaisir alimentaire pour mieux le contrôler. Ici encore, le montage, qui non seulement est fréquentiel en ce qu'il répète la scène du refus des dons, mais aussi la reprend à la fin du texte, est un signifiant.

Guelwaar, dans sa double version filmique et romanesque, déploie une disposition syntaxique qui met en échec la résignation face à un problème d'une extrême banalité, la faim. Il est 
perçu comme une fatalité en Afrique où les dirigeants se complaisent dans la distribution de dons. La nourriture permet, non seulement aux puissances impériales de maintenir les gouvernements dans le besoin, mais devient aussi un argument électoral. Les deux textes, centrés sur la nécessité de refuser l'asservissement par les dons, font de la mort du guelwaar l'élément catalyseur de cette philosophie de la dignité.

En effet, à la fin du film, lorsque le convoi des chrétiens revient avec le corps du guelwaar, ils croisent après quelques mètres de marche une camionnette transportant des sacs d'aliments. Sur le véhicule on peut lire en gros caractères: "programme alimentaire mondial». Spontanément, le jeune Étienne ordonne au chauffeur de s'arrêter. Il laisse la croix, qui va s'immobiliser entre les mains de l'abbé Léon, et entreprend de vider le contenu du véhicule sur le sol. Tous les parents, dont l'abbé et Gor Mag, sont scandalisés. Ils viennent pourtant de livrer une bataille rude afin de récupérer le corps du guelwaar mort pour avoir dénoncé l'asservissement par l'aide alimentaire. Ces réticences montrent le pouvoir corrupteur de la nourriture. Au point où Gor Mag, l'ami du défunt, implore la veuve de sommer les enfants d'arrêter "le sacrilège". Une telle rupture, une telle amnésie, est éclairante: "Ventre affamé n'a point d'oreilles", selon le dicton français. Et «l'homme qui a faim n'est pas un homme libre", de dire feu le président ivoirien Félix Houphouet Boigny.

Le plus important dans ce contexte est moins la cinglante leçon qu'administrent les enfants ou l'humiliation qu'inflige la veuve aux gérontes renégats. Le véritable sacrilège, dit-elle, est la rapide trahison de la mémoire du défunt aux côtés de qui tous les hommes avaient justement décidé de désacraliser la nourriture. Ici, la réalisation opère une répétition qui n'a pas cours dans le roman, car le récit revient sur ses traces. Après le silence embarrassé des hommes humiliés par l'action des enfants et les déclarations de la veuve, un fondu enchaîné embraie sur le discours du guelwaar, notamment sur sa partie la plus troublante:

Quant à nous, peuple sans dignité, nous dansons devant ces dons: quelle humiliation! Quand allons-nous savoir qu'une famille ne peut se bâtir, se solidifier, 
s'enraciner dans la mendicité à perpète? Et cette scène de donation se répète depuis trente ans, ici et ailleurs. Ces dons fréquemment distribués nous assassinent. Ils tuent en nous toute velléité d'orgueil, de dignité. Et ces peuplades qui nous offrent ces produits avec ostentation rient de nous chez eux.

Lorsque se termine le discours du guelwaar, un carton apparaît sur la droite de l'écran, en majuscules: "Légende africaine de l'Afrique du XXI' siècle ". Cela rappelle un peu le film muet et implique une complémentarité des langages entre l'écrit et l'écran. Au-delà de sa reprise, cette séquence apparaît comme l'argument moteur des deux textes de Sembène. Tout semble organisé dans la logique d'un récit dont la thèse préexiste à sa réalisation. Dans la perspective d'Eisenstein, le discours impliqué est compris, non pas dans les paroles d'un personnage, mais dans la dramaturgie. La structure de Guelwaar prend par cette reprise l'allure des récits et fables classiques qui se terminent toujours par une leçon ou même un message. Le cinéaste ne se veut-il d'ailleurs pas conteur? La fonction et le discours principal du conte arrivent à la fin. Dans ces textes, il s'agit de la disqualification de certaines mythologies qui tiennent l'Afrique captive de diverses formes d'aide. La véritable décolonisation, la liberté et la dignité sont dans le refus qu'assume le guelwaar au péril de sa vie. Il démonte une ruse entretenue par les politiciens : celle du Nègre affamé et jouisseur, ivre d'une philosophie gastronomique téméraire ${ }^{1}$. L'esclavage et la dépendance, quelles qu'en soient les formes, sont en soi un appel pressant à la lutte. Cela est articulé dans ce montage contre la faim, et Fanon l'écrit (1968, p. 157):

On ne peut avancer résolument que si l'on prend d'abord conscience de son aliénation. Nous avons tout pris de l'autre côté. Or l'autre côté ne nous donne rien sans, par mille détours, nous courber dans sa direction, sans, par dix mille artifices, cent mille ruses, nous attirer, nous réduire, nous emprisonner. Prendre, c'est également, sur de multiples plans, être pris ${ }^{2}$.

Le guelwaar seul le comprend, à ses dépens. En prenant gloutonnement l'aide alimentaire, l'Africain se fait prendre. La fin de 
cette mendicité est non seulement déployée par le montage fréquentatif puis par la temporalité, mais accentuée dans le film par l'ajout d'un carton. On le remarque, le discours filmique travaille de l'intérieur, à l'intérieur de l'organisation syntagmatique. Mais le film peut également signifier par les rapports qu'il instaure entre les plans ou entre les séquences.

\section{Les rapports entre plans et séquences}

Définissant le montage dans un de ses films, Eisenstein (1976, p. 108) écrit :

De fait ces images vues uniquement sous l'angle du dramatique et de l'anecdotique auraient pu être assemblées dans n'importe quel ordre. Mais sans aucun doute, la montée de la composition qui les traverse ne se serait affirmée alors dans la rigueur continue de sa construction.

Cela indique qu'aucun arrangement n'est gratuit. Mais aussi que tout signifie. Le montage tient toujours lieu de programme, explicite ou non, mais repérable dans les effets qu'il produit en ses proximités et écarts. Considérons par exemple cette prédication du catéchiste à la onzième séquence de Sango Malo: "Détournetoi de celui qui te tente, de celui qui veut t'induire en erreur. Le mal est là, le mal est là qui te guette. Mais confie-toi au Seigneur ton Dieu. Honore-le pour que tu aies la vie éternelle. Au nom de Jésus-Christ. Amen."

Or, est-ce par hasard que pendant ce sermon du catéchiste, la caméra montre en alternance Malo en train de faire son jogging dominical? La voix reste hors champ pendant que la caméra va chercher le maître qui passe juste devant ce qui tient lieu d'église. Le montage indique sans beaucoup d'ambiguïté quel est ce " mal», qui est "celui qui veut induire en erreur". Par ce raccourci, Malo et avec lui un enseignement qui récuse la foi en la Providence, le réalisateur indique ce qui est clairement élucidé dans le roman : les villageois, surtout les jeunes et les hommes, désertent le temple pour se consacrer à l'agriculture. La désertion que cette diffusion de l'athéisme provoque est grande, ainsi que le fait remarquer la caméra qui balaie la salle vide. Malo est 
un danger. Mais le discours ici est facile à repérer, si l'on compare ce montage à ceux qui régissent d'autres films.

L'Aventure ambiguë de Jacques Champreux déploie de nombreux montages secs. Des scènes de nature antithétique se succèdent, provoquant une espèce de choc chez le spectateur. Lorsque, à la huirième séquence, la Grande Royale, véritable détentrice du pouvoir dans le texte, convoque une assemblée réunissant tous les notables djallinké, il s'agit en réalité de faire le bilan de l'aventure dans laquelle elle a poussé tous les siens. Quand elle prend la parole, rompant par son timbre la lourdeur et la noblesse du chef, elle revient sur le passé glorieux, mais perdu, de son peuple. Le montage marque alors une rupture. Quand, au plan 27, elle dit: "Et plus tard, lorsque les Blancs...", sa voix qui s'amenuisait depuis les trois derniers plans disparaît totalement. Le plan suivant montre une vieille case chauffée au bois, le suivant un coucher de soleil, et le suivant encore un engin lourd en train de massacrer la nature. La Grande Royale continue, au plan 36, et termine ses propos en ces termes: «Et pourtant, il me semble que le monde est pris de vertige; que la Parole est étouffée par le fracas de leurs machines. Et notre peuple se meurt."

Cette dernière phrase est reprise par deux autres dignitaires dans le fracas des machines: le massacre de l'environnement, l'expansion de la mort sont ainsi exhaussés par le montage. À la séquence 12, une césure identique a lieu. Lorsque Samba Diallo, musulman de son état, se rend au bar pour y rencontrer le bruyant Pierre Louis, ce dernier y conduit un débat senghorien sur la fraternité interraciale. Comme pour répondre à la question de Pierre au plan 32: «Et eux [les Blancs]? Qu'est-ce qu'ils ont à nous offrir en dehors du béton et de la misère? " Le plan suivant montre une machine broyant les rochers dans un brouhaha infernal: l'asservissement de la nature et la misère à l'œuvre. On le voit avec ces articulations syntagmatiques, Jacques Champreux déploie un discours sur les désastres de l'Afrique postcoloniale, poussée au "modernisme" par l'enthousiasme de la Grande Royale. De Gobineau l'avait pourtant clairement énoncé. Dans l'expansion du "développement » et du "progrès", l'Occident "[...] travaille incessamment à s'entourer 
d'un horizon de tombes"; tout peuple qui s'y oppose "[...] est d'avance condamné et, le moment venu, détruit" (Kimoni, 1981, p. 16). La mécanique textuelle et la proximité des plans déploient le désastre.

Ces ruptures, consécutives à des scènes qui n'existent pas toujours dans le rornan, marquent le drame dans lequel se débattent les Djallinké et les autres peuples en "développement»: l'exploitation économique et le massacre de la nature. Le montage, qui coupe la parole à la Grande Royale pour alterner avec une machine en train de broyer l'environnement, expose les effets d'un choix. Pendant que les Djallinké débattent de leur sort, les Blancs continuent victorieusement leur conquête du monde. Cela rappelle fort à propos l'aveu que fait Samba Diallo à Lucienne: "Alors que l'Africain n'abat jamais un arbre sans lui adresser une prière, les Européens dévastent insolemment les forêts.» Tout cela relève d'une certaine métaphysique, d'une relation à la vie et à l'environnement. Dans un élan poétique, Frantz Fanon (1959, p. 103) avait déjà marqué ce délire de conquête:

Et voici le nègre réhabilité, [...] gouvernant le monde
de son intuition, [...] alertant les antennes fécondes du
monde, planté dans l'avant scène du monde, asper-
geant le monde de sa puissance poétique. [...] J'épouse
le monde! Je suis le monde! Le Blanc n'a jamais com-
pris cette substitution magique. Le Blanc veut le
monde; il le veut pour lui tout seul. Il se découvre le
maître prédestiné de ce monde. Il l'asservit. Il s'établit
entre le monde et lui un rapport appropriatif.

Alors qu'elle n'est perçue que très tard par les Djallinké, et notamment par la Grande Royale que Champreux montre repentante, cette fureur de conquêtes qui fait le malheur des Djallinké est pourtant annoncée par celui qu'on désigne le fou. Tout cela est rendu de manière abrupte dans un montage qui juxtapose des scènes qui n'ont pas de rapport apparent. Il s'agit d'une simple impression, car le montage est ici un signifiant, celui de la crise et, en filigrane par les confessions de Samba, de la défaite d'un peuple qui a tout misé sur un jeune garçon. Mais quand on aborde Xala on réalise que Sembène fait un autre usage du montage. 
Dans Xala, la dramaturgie est beaucoup plus l'expression d'une opposition presque manichéenne: riches/pauvres, vertueux/crapules. Il est à cet égard significatif que le régiment de gueux y apparaisse toujours en face de El Hadji. La disposition est celle de l'affrontement. En plus, ils prennent toujours position non loin de lui, le suivent dans toutes ses pérégrinations. Il s'agit là d'une mise en opposition de la misère et de l'opulence malsaine et, dans une certaine mesure, de la vertu et du vice. Il est à cet égard édifiant qu'une ménagère vienne deux fois verser le contenu puant de son récipient devant l'égout situé juste devant la boutique de El Hadji, le séparant ainsi du peloton d'aveugles dont la musique hante tout le film. Ne doit-on pas faire un rapprochement entre la pourriture déversée et le déclin ayant provoqué ce xala qui afflige l'Afrique? L'eau sale ne rejoint-elle pas ainsi fatalement sa source ou, tout au moins, un lieu symbolique?

Que dire en outre de cette carte de l'Afrique derrière El Hadji, montrée lors de la visite de sa fille Rama dans le bureau? Cette carte est veinée de frontières coloniales, alors que la frontière derrière Rama ne porte les traces d'aucune violence. Au-delà du face à face au cours duquel la fille domine spatialement le père, presque coupé par la caméra et coincé à la gauche de l'écran, ne fautil pas aussi lire dans la fissuration du continent l'éclatement de toute vertu dont le père de Rama est la triste illustration?

Ces exemples le montrent, le montage porte le film à une dimension sémantique que le roman ne permet pas d'envisager. Étant donné que ces éléments n'existent pas dans le texte littéraire, l'ajout du texte implique donc presque toujours un ajout de sens. Bien plus, le lien et le rapprochement entre deux plans, la mise en scène même provoquent toujours une espèce de choc duquel naît une plus-value sémantique. La rupture narrative a ici, écrit Marcel Martin (1985, p. 152), une valeur symbolique, mais surtout expressive:

[...] il y a le montage expressif, fondé sur des juxtapositions de plans ayant pour but de produire un effet précis par le choc de deux images; dans ce cas le montage vise à exprimer par lui-même un sentiment ou une idée ; il n'est plus alors un moyen mais une fin: loin d'avoir 
pour but idéal de s'effacer devant la continuité en facilitant au maximum les liaisons les plus souples d'un plan à l'autre, il tend au contraire à produire sans cesse des effets de rupture dans la pensée du spectateur, à le faire trébucher intellectuellement pour rendre plus vivace en lui l'influence de l'idée exprimée par le réalisateur et traduite par la confrontation des plans.

On se situe dans la droite ligne du "montage des attractions" développé par Eisenstein. L'apparente innocence des plans, leur mise en scène, participent en fait d'une stratégie discursive dont il importe de déceler les signifiés. Pour tous les films considérés ici, ils sont presque les mêmes : l'engagement pour la liberté, la défense de ce qui reste d'une dignité menacée par des décennies de corruption et de misère. Mais l'idéologie et le discours peuvent aussi naître de ce qui est montré, de la manière de le montrer. On se rend compte, à ce sujet, que les manœuvres descriptives de la caméra sont tout aussi éloquentes. Il y a donc, en plus de celui du montage, un discours des figures.

\section{Caméra et manœuvres descriptives}

La conduite des récits littéraires est difficilement imaginable sans des passages descriptifs. Si on admet que le film est récit, il est donc légitime d'y envisager une fonction des descriptions. Selon Christian Metz (1968, p. 28),

[...] un grand nombre de récits contiennent des descriptions, et il n'est même pas certain qu'on puisse trouver des descriptions ailleurs qu'en enclaves dans les récits. Ainsi la description apparaît à la fois comme l'opposé de la narration et comme une des grandes figures ou un des grands moments de la narration. Ce mélange curieux d'antinomie et de parenté, par quoi se définissent intuitivement les rapports de la narration et de la description, se laisse mieux élucider si l'on fait entrer darıs le "système" un troisième terme, l'image : narration et description s'opposent en commun à l'image parce que leur signifiant est temporalisé, alors que celui de l'image est instantané $[. . .]^{3}$.

Si le nouveau roman a fait de la description un enjeu théorique, la question est tout autre dans les réécritures filmiques 
considérées dans cette étude. Tout ce qui est noté étant notable, comme le dit Roland Barthes, l'élément moteur de la description que définit Metz, à savoir l'image, mérite d'être considéré dans sa nature, son contenu, sa fréquence, les moments et les modes de son apparition. Les projets idéologiques variant avec les réalisateurs, il n'est dès lors pas étonnant que les traitements descriptifs le soient également.

La plupart des plans et séquences de description opèrent sous une forme expositionnelle. La caméra, comme innocemment, balaie une surface et peut s'y attarder plus ou moins longtemps. Ce n'est plus un oil, mais un regard. Ainsi, quand dans Sango Malo le maître découvre sa "demeure", un lent panoramique la parcourt pour en offrir l'allure au spectateur et au nouvel occupant: des bestioles circulant allégrement dans la pièce, un vieux lit, un sol jonché de tous genres de déchets, etc.

Dans Sarraounia, dès la septième séquence est montré un village après le passage de troupes coloniales. Le tableau est désastreux : arbres et maisons incendiés, nombreux débris en flammes, cadavres jonchant un sol craquelé. La caméra s'attarde quelques instants sur un bébé se débattant douloureusement auprès du corps de sa mère égorgée. La même affiche se répète à la séquence suivante: un vieux chef mendie la clémence des mercenaires pour sauver son village du désastre. Le capitaine Voulet, chef de l'expédition, lui flanque une balle dans la tête et sa bande armée se jette à l'assaut de la bourgade. La caméra assume la même fonction que précédemment. Elle devient plus qu'un regard: un témoignage. Très lentement, elle montre les images du désastre: incendies géants, cadavres disséminés sur le sol, têtes tranchées avec acharnement par des cavaliers. Tout au long de ces deux séquences, l'image prime. Le son est produit par la flûte dont la mélodie, tragique et mélancolique comme celle du mendiant dans Xala, accompagne ces images de détresse et de violence. Vers la fin du film, après la bataille décisive et coûteuse contre Sarraounia, Voulet contraint les soldats à continuer la route. Le même cynisme colonial est mis en scène: les soldats blancs achèvent les blessés à coups de pistolet. Un plan rapproché, laid et touchant, insiste sur l'agonie d'un soldat nègre se tordant d'une douleur qui l'emportera après le coup de plomb 
de son maître. Rien de cela n'est accidentel, qui relève d'un projet, d'une idéologie. Alain Garcia (1990, p. 68) écrit:

Décrire, c'est faire preuve d'un parti pris, c'est décider de faire un choix et d'accorder de l'importance (du temps et de l'espace) à quelque chose ou à quelqu'un. D'un point de vue idéologique, c'est prendre position et afficher des intentions, ceci tant en littérature qu'au cinéma. [...] Si tout ce qui est décrit est important et susceptible d'avoir de la valeur, ce qui n'est pas décrit est généralement aussi du point de vue idéologique et peut parfois se révéler supérieur dans la diégèse.

On peut l'observer dans les cas précédents, les procès descriptifs accompagnant le récit sont principalement dus aux panoramiques et travellings, ralentis par la réalisation. La musique funèbre les accompagnant est plus éloquente que quelque discours oral. Il ne reste plus au spectateur qu'à construire l'intelligibilité de cet espace-temps rare dans les cinémas dominants. Mais l'activité descriptive peut non moins être inférée de la position de la caméra ainsi que de la distance focale.

L'observation des réécritures filmiques qui nous occupent révèle un cas pour le moins troublant. On serait tenté de dire de L'Enfant noir qu'il s'agit d'un documentaire qui ne dit pas son nom. On remarquera que, de tous les films retenus dans cet article, aucun ne privilégie les contre-plongées, et surtout pas les plongées dont le côté réducteur est avéré, du moins dans le cinéma occidental: "La plongée en effet (prise de vue de haut en bas) a tendance à rapetisser l'individu, à l'écraser moralement en l'abaissant au niveau du sol, à faire de lui un objet englué dans un déterminisme insurmontable, un jouet de la fatalité" (Martin, 1985, p. 44). Dans L'Enfant noir, certaines conversations entre le petit Baba et son père, les gisements d'or et leur exploitation ainsi que les transactions silencieuses au début du film, le vieux taxi, tas de ferraille en train de se déplacer vers Conakry, certaines rues de la ville avec ses caniveaux sales, tout est montré en plongée. La cérémonie des secondes noces de Moussa n'existe pas dans le roman où, d'ailleurs, l'oncle de Camara, déjà polygame au moment où il arrive, mène une vie paisible. Mais elle est filmée alternativement en plongée selon le point de vue de la 
première épouse qui regarde la cérémonie de l'étage où est situé l'appartement. L'emploi de la plongée est ici pour le moins paradoxal et semble relever d'une espèce d'ironie. La préférence monogarne est quand même située en altitude, alors que les larmes de la première épouse indiquent que l'institution que la caméra semble placer en position de pouvoir est en détresse. Le paisible ménage romanesque est gravement en crise et menacé d'un regard panoptique, quoique pathétique, dans le film.

Le discours latent est facile à déchiffrer: la polygamie est d'une platitude certaine, sinon un danger. La réécriture filmique dévoile ainsi les motivations mal dissimulées par une précision du générique: "Ce film s’inspire librement du récit autobiographique L'Enfant noir écrit par Camara Laye en 1953. " Si la réécriture a pour fondement la liberté du créateur et "l'ouverture" de l'œuvre, on doit également admettre, nous dit Umberto Eco, qu'il y a "une façon de représenter la réalité qui devient discours et jugement [...]» (1965, p. 155). Autrement dit, on passe fatalement, dans le cas de Laurent Chevalier, de la représentation, de la réécriture, à la perception et à l'énonciation d'un discours dominant: la vérité monogame triomphante en Occident, conquérante en Afrique. On pourrait, dans ce cas, avancer l'hypothèse de Gabriel H. Teshome sur Jean Rouch au sujet de ses films "sur l'Afrique». Ignorant, le public occidental serait l'otage d'une réalisation qui se révèle, peut-être sans le savoir, l'instrument d'un impérialisme culturel. Le film en dit davantage sur le réalisateur que sur l'Afrique (Teshome, 1982, p. 79), mais aussi porte le roman vers un discours nouveau.

En plus des plongées, les variations scalaires relèvent aussi d'une certaine volonté descriptive et discursive. Est-ce un hasard si dans Sango Malo la caméra est braquée sur les pieds sales ou sur les livres déchiquetés d'un jeune élève? La démarche est identique chez Chevalier: le contenu sale des calebasses contenant des graines d'or, le visage du commerçant Traoré, les mains crasseuses et noires comptant les billets, le repas incroyablement maigre servi à Baba Camara par sa mère, les cheveux crépus de Baba qui restent dans le même état, pendant presque tout le film ${ }^{4}$, les jouets et les instruments de la forge, tout est présenté en plan rapproché ou en gros plan. Ces fragments du corps semblent 
ainsi connoter sa démolition. Mais lorsque après une mésaventure, Baba est contraint de rentrer chez lui à pied, il est montré errant dans les rues de Conakry. Toutefois, ce n'est pas exactement lui qu'on voit, il est presque perdu dans l'espace. Il est égaré dans le cadre, à l'arrière-plan duquel on voit des vieilles carcasses de voiture et des tas d'immondices. La réalisation opère ici un usage ouvertement idéologique pour offrir un discours inapparent: l'Afrique, et cela est facile à retenir, est un catalogue de misères et de crasse.

Ousmane Sembène use aussi du gros plan qui a chez lui, beaucoup plus que chez Chevalier, valeur de synecdoque et de métonymie. Dans Xala, lorsque le gang fraîchement constitué des "hommes d'affaires" revient pour assister au mariage, des gros plans successifs montrent leurs mallettes, indices d'une classe sociale pour l'objet et métaphores de la corruption par leur contenu. Au lieu de montrer les hommes, la caméra s'attarde sur des objets qu'ils portent. La figure synecdochique semble bien opérante. Linsistance sur ces mallettes métaphorise la perversion originelle des indépendances, leur accorde une fonctionnalité et porte le texte filmique jusqu'à un horizon inexploré par le roman. On ne s'en rend compte qu'à la fin: Thielli, qui n'existe pas dans le roman, prend la place de El Hadji et reçoit immédiatement la petite valise que portait le commerçant. L'objet et ses attributs changent de propriétaire. On assure ainsi la continuité de la déchéance morale. Tel est le portrait d'une bourgeoisie africaine qui, comme Fanon l'avait déjà entrevu, se trouve réduite à la combine.

Med Hondo quant à lui est constant dans la dérision à laquelle il soumet le néocolonialisme. Après la révolte de Baka, où l'amante reine accorde ses faveurs à Gogué, la voix off s'empare de l'autorité narrative à la cinquième séquence et explique: "À la fin du XIX siècle, à Berlin, les puissances occidentales s'étaient partagé l'Afrique: Français, Anglais, Allemands, Espagnols, Italiens et Belges investissaient le continent africain. » Mais pendant que ce discours est livré, la carte de l'Afrique est l'objet d'un gros plan. Elle se fissure progressivement sur l'écran, assaillie de tous côtés par les flammes. Une espèce d'onde déconstruit la carte qui tourne au rouge. Suit l'image d'un vieux s'écroulant 
sous les balles, puis un sol craquelé de toutes parts auquel succède encore la même carte zébrée de frontières sanguinolentes. Elle se rapproche encore davantage de la caméra. Suit alors la colonne assassine du capitaine Voulet. On se trouve devant le discours favori de Med Hondo: la violence impériale, l'affreuse charcuterie de Berlin érigeant des murs en Afrique alors que les Européens s'évertuent à supprimer les leurs.

L'opération de Hondo est d'autant plus importante qu'elle relève d'une mise en scène, d'un truquage, car l'image n'est pas "vraie». Relever l'intérêt de ce rapport métaphorique entre truquage filmique et truquage colonial, éternel. C'est ce qui est entrevu: le néocolonialisme, comme le cinéma, est truquage, mise en scène, planification, mais surtout calcul assassin. C'est ainsi qu'il triomphe depuis des siècles. On le voit, la seule image, son organisation interne, son contenu, tout comme ses agencements, sont des formes d'élaboration d'un discours qu'on percevra difficilement, mais qui existent avec l'image.

\section{Conclusion}

Largument principal de cet article est que les réécritures filmiques n'ajoutent pas du sens seulement en annexant des éléments absents du texte littéraire. Le début et la fin d'un film sont des lieux stratégiques. En plus des discours du montage, ceux de la description sont aussi repérables. Le texte littéraire devient ainsi une banque de données dont se sert le réalisateur pour explorer des horizons sémantiques inapparents. Les usages de l'appareillage technique ainsi que l'ordonnancement des images permettent de garantir une plus-value dont l'enjeu varie avec le signataire de l'œuvre. Mais les Africains semblent constants dans le démantèlement de toute idéologie impériale ou de toute forme d'aliénation.

University of Calgary

\section{NOTES}

1. Se souvenir, à ce sujet, du schéma un peu réducteur par lequel Glauber Rocha et Lino Micciche enferment les débuts du "cinéma novo" brésilien dans ce qu'ils nomment " une esthétique de la faim». Le second en arrive même à la conclusion que les 
pays sous-développés comptent très peu ou pas de philosophes pour la simple raison que les problèmes de la chair enchaînent la faculté de penser. A propos de cette simplification, voir le texte de Christian Zimmer (1974, p. 206-209) intitulé "Culture et pauvreté".

2. C'est l'auteur qui souligne.

3. C'est l'auteur qui souligne.

4. Une seule exception: avant le mariage, Baba se fait couper les cheveux en même temps que son oncle. Hypothèse : cette scène a été tournée après cette cérémonie, puis montée suivant cette organisation séquentielle. Sinon, à aucun moment du film, les cheveux du jeune broussard ne sont peignés. Hasard?

\section{OUVRAGES CITÉS}

Benveniste, Émile. Essais de linguistique générale. Paris : Gallimard, 1966.

Colin, Michel. Langue, film, discours. Prolégomènes à une théorie générative du film. Paris: Klincksieck, 1985.

Comolli, Jean-Louis. "Technique et idéologie I. Caméra, perspective, profondeur de champ". Cahiers du cinéma, n' 229, p. 4-21.

Comolli, Jean-Louis. "Technique et idéologie II. Caméra, perspective, profondeur de champ ". Cahiers du cinema, n' 230 (1971), p. 51-57.

Comolli, Jean-Louis. "Technique et idéologie III. Caméra, perspective, profondeur de champ ". Cahiers du cinéma, no 231 (1971), p. 42-49.

Comolli, Jean-Louis. "Technique et idéologie IV. Caméra, perspective, profondeur de champ". Cahiers du cinéma, nos 234-235 (1971), p. 39-45.

Eco, Umberto. L'CEuvire ouverte. Paris : Seuil, 1965.

Eisentein, Sergeï M. Le Film : sa forme, son sens. Paris : Christian Bourgois, 1976.

Fanon, Frantz. Les Dainnés de la terre. Paris: Maspéro, 1968.

Fanon, Frantz. Peaux noires, masques blancs. Paris: Seuil, 1959.

Foucault, Michel. Les Mots et les choses. Paris: Gallimard. 1966.

Garcia, Alain. L'Adaptation. Du roman au film. Paris: Diffusion Dujaric, 1990.

Kimoni, Iyay. Une image du Noir et de sa culture. Neuchâtel: H. Meisseiller, 1981.

Martin, Marcel. Le Langage cinématographique. Paris: Cerf, 1985.

Metz, Christian. Le Signifiant imaginaire. Paris : UGE, 1977.

Metz, Christian. Essais sur la signification au cinéma. Paris : Klincksieck, 1968.

Teshome, H. Gabriel, Third Cinema in the Third World. The Aesthetics of Liberation, Ann Arbour: UMI Research Press, 1982.

Zimmer, Christian. Cinéma et Politique. Paris: Seghers, 1974. 